\title{
Motivaciones que inciden en la participación de docentes en procesos de educación para la sexualidad
}

\author{
Motivations affecting the participation of \\ teachers in sex education processes
}

Lucila Niño-Bautista $^{1}$; María del Pilar Oviedo-Cáceres ${ }^{1}$; María-Constanza Hakspiel-Plata ${ }^{1}$; Claudia-Milena Velasco-Rangel ${ }^{1}$;

Diana-Carolina Galvis-Padilla ${ }^{1}$ Darlen Aragón-Borré1; Liliana-Patricia Pineda-Rodríguez ${ }^{1}$

Forma de citar: Niño Bautista L, Oviedo Cáceres MP, Hakspiel Plata MC, Velasco Rangel CM, Galvis Padilla DC, Aragón Borré D, et al. Motivaciones que inciden en la participación de docentes en procesos de educación para la sexualidad. Rev Univ Ind Santander Salud. 2018; 50(2): 144-156. doi: http://dx.doi.org/10.18273/revsal.v50n2-2018006 @ @ (i)

\section{Resumen}

Objetivos: Determinar las características de la motivación y comprender los significados que tiene, para los colectivos docentes, participar en procesos formativos en educación para la sexualidad. Metodología: Método mixto: etapa cuantitativa fundamentada en la teoría de Deci y Ryan, evaluó motivación interna, externa y desmotivación, utilizando el modelo de Rasch; etapa cualitativa con enfoque fenomenológico y aplicación de entrevistas semiestructuradas. Resultados: Participaron 104 docentes de siete instituciones educativas del municipio de Socorro - Santander. El análisis sobre ítems y docentes, según modelo Rasch, para los tres tipos de motivación, tuvo buenos resultados que permitieron identificar las características relacionadas, así como determinar que la motivación interna fue la que llevó a la mayoría de docentes a participar en el proceso formativo de educación para la sexualidad. Esto fue corroborado por las entrevistas, evidenciando que el colectivo docente interpretó dicho proceso como una oportunidad para mejorar su práctica docente y aumentar sus conocimientos en el tema. Conclusiones: La ubicación del $66 \%$ de docentes en lo alto de la escala de motivación interna permitió identificar la fuerza motivacional para asistir al proceso formativo. Atender a intereses y necesidades, así como reforzar la autonomía y la autodeterminación en grupos docentes que requieren capacitación en educación para la sexualidad, es una buena forma de lograr participación y compromiso estable y duradero como formadores en el área. De acuerdo con la búsqueda bibliográfica realizada, este estudio sobre la motivación y los procesos de capacitación docente en educación para la sexualidad es pionero.

Palabras clave: Motivación; Encuesta; Docentes; Educación; Sexualidad.

\begin{abstract}
Objectives: To determine the characteristics of motivation and to understand the significance for teachers, related to participation in sex education training processes. Methodology: Mixed method: quantitative phase based on the theory of Deci and Ryan, internal and external motivation, and demotivation were assessed using the Rasch
\end{abstract}

1. Universidad Industrial de Santander. Bucaramanga, Colombia.

Correspondencia: Lucila Niño-Bautista. Dirección: Carrera 32 n. 29-31. Teléfono: +57 764500 06. Correo electrónico: lucinino@hotmail.com 
model; qualitative stage, with a phenomenological approach, included the application of a semi-structured interview. Results: 104 teachers from seven educational institutions in Socorro-Santander participated in the study. Analysis of items and teachers, according to the Rasch model for the three types of motivation, had good results that allowed us to identify the related characteristics and to determine that internal motivation led, on the majority of teachers, to participate in the formative process of sex education. This was corroborated by the interviews, where teachers interpreted the process as an opportunity to improve their teaching practice and increase their knowledge on the subject. Conclusions: The allocation of $66 \%$ of teachers at the top of the internal motivation scale allowed us to identify the motivational force to attend the formative process. To respond to interests and needs, as well as to reinforce autonomy and self-determination in educational groups that require training in sex education, is a good way to achieve stable and lasting participation and commitment as trainers in the area. Based on our bibliographic search, this study on motivation and the processes of teacher training in sex education is a pioneer.

Keywords: Motivation; Schools; Teacher training; Sexuality; Personal autonomy.

\section{Introducción}

La formación permanente de docentes constituye uno de los principales factores dinamizadores y calificadores de los sistemas educativos. Diferentes diagnósticos acerca de los mejores aprendizajes logrados por escolares y el incremento de la calidad de la educación, subrayan la importancia del profesorado como actores centrales en el proceso educativo ${ }^{1-3}$.

Una de las áreas de interés en la cualificación docente en educación básica y media en Colombia tiene que ver con la educación para la sexualidad y la construcción de ciudadanía, dado el alto impacto que estas competencias, desarrolladas oportuna y adecuadamente en niñas, niños, adolescentes y jóvenes, tienen en el desarrollo individual y social de las nuevas generaciones y del país como un todo ${ }^{4}$; la efectividad del trabajo para formar personas autónomas, responsables y respetuosas de los derechos humanos además de competentes profesionalmente, se evidencia en estudios realizados por la OCDE (disponibles en su página Web), en países que le han apostado a la educación de calidad como política de Estado, así mismo en quienes han evaluado avances y factores causales para la mejora de la calidad educativa, identificando que la participación docente en los cambios propuestos, el trabajo en red, el involucramiento de la comunidad y la reconsideración del liderazgo, entre otros, han sido fundamentales ${ }^{5}$.

En Colombia a partir de 2005 el Ministerio de Educación Nacional en asocio con el Fondo de Población de Naciones Unidas UNFPA, crearon el programa de Educación para la sexualidad y construcción de ciudadanía como una apuesta ética y política, que puede agenciar escenarios más equitativos y participativos desde la afirmación de la diversidad y la diferencia. En el informe de evaluación del programa en 2008, directivas y docentes hablaron sobre sus beneficios y las dificultades afrontadas, siendo en general positiva, y esperanzadora la experiencia vivida; sin embargo, al referirse a su rol docente frente a la sexualidad, expresaron vulnerabilidad por tener que hablar con estudiantes sobre cosas para las cuales ellos aún no están preparados ${ }^{6}$.

Este temor del colectivo docente de asumir la educación para la sexualidad y la ciudadanía ha sido conocido de primera mano por el Instituto PROINAPSA de la Universidad Industrial de Santander, pues en su trabajo de más de 12 años en la formación en el tema con docentes y líderes estudiantiles en el departamento, la región y el país, así lo ha observado ${ }^{7,8}$. Estos temores del personal docente, de muy variado origen y manifestados en diversas formas, pueden generar barreras para su proceso de capacitación y constituirse en obstáculos para asumir la educación para la sexualidad como parte de su rol. Dichos temores y resistencias han sido identificados en otras investigaciones, así como la forma en que esta población afronta sus dudas, desconocimientos y mitos acerca de la sexualidad cuando deben asumir la educación de sus estudiantes, con quienes tienen diferencias generacionales y culturales ${ }^{9}$.

Investigaciones realizadas en Costa Rica ${ }^{10}$, Brasil ${ }^{11}$, Venezuela $^{12}$, Argentina ${ }^{13}$ y Estados Unidos ${ }^{14}$ coinciden en algunos puntos clave: la inseguridad de docentes al sentir que no tenían el conocimiento apropiado o suficiente para hacer educación para la sexualidad; la influencia de los propios miedos, tabúes y culpas frente a la sexualidad, y la dificultad para hablar abiertamente sin sentir que serían juzgados. Así mismo, algunos estudios coincidieron en que los docentes no necesitaban ser expertos en educación sexual: básicamente, se debían crear espacios de formación docente donde se pudiera contar con un talante abierto para facilitar el 
diálogo y dejar de lado el abordaje de esta materia de forma dogmática, además, contar con herramientas que les facilitaran crear contextos educativos adecuados.

Como varios autores lo han identificado y como ha sido la experiencia del Instituto PROINAPSA UIS, se puede empoderar al profesorado para orientar asertivamente a sus estudiantes en la educación para la sexualidad. Sin embargo, uno de los retos que persiste es conseguir su interés, apertura mental y disposición desde los primeros contactos, pues de esa manera podrían obtener mejores resultados para llevar a cabo su trabajo con los estudiantes. Por ello, se decidió estudiar la motivación de los grupos docentes que van a participar en procesos de educación para la sexualidad, basados en la potencia explicativa y predictiva de ésta en la conducta humana como lo plantearon Manassero y Vázquez ${ }^{15}$.

El manejo de la motivación de docentes en procesos de educación para la sexualidad en el Instituto PROINAPSA UIS se ha fundamentado en la teoría de Deci y Ryan ${ }^{16}$, llamada también de la autodeterminación - TAD. Esta plantea que las conductas humanas se dan con un grado variable de auto determinación, por lo cual pueden ser precedidas de un proceso de reflexión que lleva a las personas a elegir en forma voluntaria y a comprometerse con lo que han elegido. Este planteamiento, además de mostrar mayor concordancia con los procesos internos de decisión de personas adultas, se fundamenta en la necesidad de autonomía y estimula la metacognición en docentes para reflexionar y aprender sobre fundamentos teóricos en que se basan los procesos de formación en derechos y deberes sexuales y reproductivos y construcción de ciudadanía, para interactuar con estudiantes en el escenario educativo.

De acuerdo con Deci y Ryan, al convocar equipos docentes para participar en cursos de educación para la sexualidad, se ponen en movimiento fuerzas de origen psicológico que hacen interactuar dos dimensiones que operan en el proceso motivacional: la intrínseca o interna, ligada a los intereses, deseos y expectativas y la extrínseca o externa, entendida como los aspectos del contexto que pueden funcionar como estímulo; también se puede presentar la ausencia de motivación ${ }^{17}$. En los tres casos difiere el grado de autonomía y autodeterminación; Balaguer, et al. citando a Deci, plantearon que las conductas intrínsecamente motivadas son el prototipo de las decisiones autodeterminadas ${ }^{18}$. En el caso de la motivación externa, un meta análisis de 128 investigaciones comprobó que cuando las personas deciden porque hay estímulos monetarios $\mathrm{u}$ otros estímulos contingentes asociados a la conducta, estos socaban la motivación interna y el locus de causalidad pasa de lo interno a lo externo. En la no motivación los sentimientos de autodeterminación no existen y no se dan razones ni extrínsecas ni intrínsecas que sustenten la conducta ${ }^{19}$. En la TAD las necesidades son los mediadores psicológicos que influyen en los tres tipos de motivación y a su vez en la personalidad y lo afectivo, entre otros ${ }^{20}$.

Vallerand, et al. ${ }^{21}$, elaboraron y validaron una encuesta en 1989 para operacionalizar la TAD; la encuesta, construida inicialmente en francés y posteriormente traducida al inglés y al español, tuvo buenos resultados psicométricos en sus tres versiones en concordancia con los planteamientos teóricos de Deci y Ryan. Dicho instrumento ha permitido a diferentes investigadores el desarrollo y validación de otras encuestas, las cuales fueron referentes para este estudio ${ }^{22}$.

Otras corrientes más recientes, han propuesto un camino diferente para lograr procesos de aprendizaje exitosos y sincronizados con el contexto de hoy y las dimensiones objetivas de la vida y el pensamiento de las nuevas generaciones; han pasado de la autodeterminación a la autorregulación, fundamentada en la psicología cognitiva y referida al desarrollo de una capacidad esencial para que estudiantes de educación básica, media y superior tengan éxito académico ${ }^{23}$. Existen varios modelos que cuentan con elementos comunes y énfasis en distintos aspectos. Paul Pintrich, define el aprendizaje autorregulado como un proceso activo-constructivo en el cual la persona fija sus propias metas de aprendizaje, monitorea, regula y controla su adquisición de conocimientos, motivación y conductas ${ }^{24}$. De igual forma, la teoría de metas de logro que surge de la investigación de varios autores (Ames, Archer, Dweck, Elliot, Legget, Maher y Nicholls entre otros), identifica dos perspectivas: de orientación a la tarea y de orientación al ego. En la primera el éxito viene por el dominio de la tarea y el progreso personal; en la segunda, el éxito viene por la victoria ante las personas rivales y demostración de mayor capacidad que ellas. Zimmerman considera la autorregulación, como un proceso interactivo que implica no solo habilidades de conducta para manejar las contingencias ambientales, sino el conocimiento y un sentido de autoeficacia (personal agency) para activar esas habilidades en contextos relevantes. Boekaerts y Corno definen dos tipos de autorregulación: a) Top down, que se inicia en los intereses, valores y metas de la persona y b) Bottomup, en la que se regula por criterios o guías externas que la persona asume ${ }^{25-27}$. 
Järvela y Järvenoja, conceptualizan la autorregulación como un fenómeno dual individual-social, en la cual, en un ambiente colaborativo de aprendizaje las personas de un grupo representan agentes interdependientes, autorreguladores y simultáneamente constituyen una entidad social que crea posibilidades de aprendizaje para el $\mathrm{mismo}^{28}$.

Finalmente, se consideró que la presente investigación sería un buen aporte para seguir construyendo, junto a otros estudios que se continuarán realizando, mejores y más interesantes intervenciones educativas en el área; así mismo, se continuará incrementando las habilidades de equipos formadores de docentes en educación para la sexualidad, con el fin de interactuar con personas reacias a trabajar el tema, ayudándolas a mejorar el manejo de sus negaciones y temores para participar en estos procesos, obteniendo así a mediano y largo plazo, mayor efectividad en la formación de colectivos docentes en el tema.

Como objetivos del estudio se definieron: 1) Analizar las características de la motivación de docentes que participaron en un proceso de educación para la sexualidad. 2) Comprender desde la perspectiva de integrantes del colectivo docente, las motivaciones para participar del proceso formativo en educación para la sexualidad.

\section{Materiales y métodos}

En el estudio participaron docentes de siete instituciones públicas -urbanas y rurales- de educación básica y media del municipio de Socorro - Santander. El colectivo docente recibió 30 horas de capacitación sobre educación para la sexualidad, por el grupo de profesionales del Instituto PROINAPSA UIS, e igualmente asesoría y asistencia técnica durante su implementación con estudiantes a lo largo del año escolar. Para la fase de investigación, al final del proceso de capacitación se trabajó con docentes que aceptaron la invitación a participar, por lo cual la muestra fue de conveniencia.

\section{Método}

En el proceso investigativo se utilizó el método mixto cuantitativo - cualitativo siguiendo el diseño combinado secuencial explicativo ${ }^{29,30}$ (Figura 1). En la etapa cuantitativa se evaluó motivación interna, externa y desmotivación en el grupo docente; en la cualitativa se seleccionaron algunas personas de cada grupo según tipo de motivación (modelo de selección de participantes), quienes aportaron información que permitió expandir y enriquecer la interpretación y comprensión de los resultados de acuerdo con el contexto en que los docentes del estudio ejercían su rol.

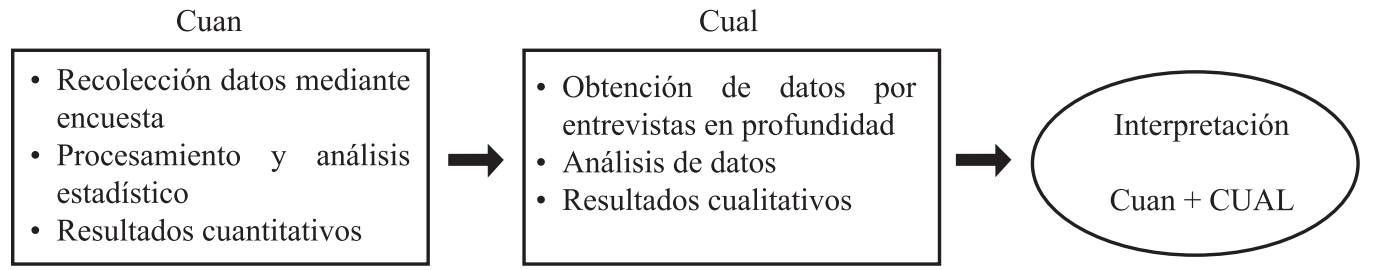

Figura 1. Diseño combinado secuencial explicativo - Creswell, et al.

\section{Instrumentos y técnicas de recolección de datos}

Para la etapa cuantitativa se utilizó instrumento tipo encuesta previamente construida por el grupo, en el marco del modelo TAD de tres categorías y siete sub categorías. Los resultados del proceso de validación de la encuesta (aparente y de constructos), fueron publicados en artículo separado ${ }^{31}$. La encuesta está conformada por dos secciones: la primera con 10 variables socio demográficas y culturales (edad, sexo, estado civil, hijos u otros menores bajo su cuidado, nivel educativo, área del conocimiento en título de pregrado, motivación para ser docente, años de experiencia docente, religión profesada) y la segunda con 56 ítems de las tres categorías y respectivas subcategorías sobre motivación con opción de respuesta dicotómica (sí, no).
Para el abordaje cualitativo, se utilizó el enfoque fenomenológico, utilizando como técnica de recolección de información entrevistas semi-estructuradas y para su análisis el modelo propuesto por Taylor y Bogan ${ }^{32}$. Las entrevistas se programaron para seis docentes que habían diligenciado la encuesta y buscaron ahondar mediante la conversación, los temas de interés captando las vivencias, opiniones y concepciones que tuvieron al momento de la convocatoria para participar en el proceso formativo en educación para la sexualidad, y lo ocurrido en términos de motivación durante el proceso de capacitación; se formularon preguntas como: ¿Cuáles fueron los sentimientos que tuvo al momento de recibir la invitación a participar en el proceso formativo?, ¿Cuál fue esa primera sensación cuando le llamaron a hacer parte de un proceso de formación en sexualidad?, 
¿Cuáles fueron las motivaciones que le llevaron a estar en el proceso?

\section{Procedimiento, recolección y análisis de datos}

En la etapa cuantitativa una vez se concluyó la capacitación, el grupo docente fue invitado a diligenciar la encuesta en horario laboral para facilitar su participación; recibió información sobre el objetivo de la investigación, la condición de anonimato y el manejo responsable y riguroso de la información recopilada; además a cada persona se le solicitó consentimiento informado de manera verbal y a quienes optaron por no participar, se les respetó su decisión. Se instruyó sobre la estructura de la encuesta y el necesario proceso de lectura reflexiva que implicaba responder los ítems sobre motivación. Terminada la recolección de los datos, se aplicó el protocolo para depuración de la base de datos (doble digitación en Excel por diferente persona, correcciones de errores detectados e impresión de base de datos definitiva) y se transfirió a los paquetes estadísticos Stata v 10.0 y Winstep 3.81 para su análisis. Para descripción de las variables sociodemográficas se utilizaron proporciones y promedios; en los ítems de motivación, los estadísticos del modelo de Rasch -infit, outfit, confiabilidad, separación, varianza por medidas y varianza inexplicada en primer contraste- para definir ajuste al modelo. Se decidió utilizar el modelo de Rasch, por las ventajas que tiene sobre la teoría clásica de los test - TCT como realizar la medición conjunta de los parámetros tanto de personas como de ítems, ajustar datos al modelo sin interdependencia entre ítems y personas, las propiedades de intervalo de la escala de medición, el análisis de validez del constructo y la medición de la habilidad de la persona al responder los ítems ${ }^{33}$. Para establecer significancia estadística entre las variables sociodemográficas y los tipos de motivación se utilizaron los promedios de las medidas en la prueba $\mathrm{T}$ de Student y en la prueba exacta de Fisher para tablas de contingencia, la ANOVA de una vía para variables con más de dos grupos, la prueba de Bonferroni para ajustar por múltiples comparaciones y la prueba de MantelHanzel para identificar funcionamiento diferencial del ítem-FDI por sexo.

Para la etapa cualitativa, las entrevistas se realizaron de manera individual y se buscó ahondar con las personas participantes los significados relacionados con los tipos de motivación y su participación en el proceso de formación. Para el análisis de las entrevistas realizadas se siguió el proceso de descubrimiento, codificación y relativización de los datos y para apoyar el análisis de los relatos se utilizó el software ATLAS ti (v 6.2) Qualitative data analysis.

\section{Resultados}

Participaron en el estudio de manera voluntaria 104 docentes equivalentes al $51 \%$ de quienes habían iniciado su proceso formativo un año antes del diligenciamiento de la encuesta.

\section{Características sociodemográficas}

El rango de edad estuvo entre 26 y 65 con promedio en 47 años; el $81 \%$ del grupo eran mujeres, el $77 \%$ vivía en pareja (casados y en unión libre) y el 53\% tenía hijos menores de 18 años; la religión predominante fue la católica con el $90 \%$. El área de titulación de pregrado se distribuyó así: ciencias de la educación $(53,8 \%)$, ciencias sociales y humanas $(13,4 \%)$ y matemáticas y ciencias naturales $(11,5 \%)$, y otras $21,4 \%$. En estudios de posgrado, el $78 \%$ tenía especialización y el $10,5 \%$ maestría; solo el $10 \%$ de las personas terminó siendo docente porque no encontró otra opción laboral.

\section{Etapa cuantitativa}

Como se indicó, en esta etapa se utilizó el modelo de Rasch y dado que una de sus características en el análisis es la unidimensionalidad se estudió por separado la motivación interna, la externa y la desmotivación (Tabla 1).

Previo al análisis se excluyeron: en MI cinco personas que no ajustaron al modelo, en ME tres personas y cuatro ítems y en DESM dos personas que respondieron a todo Sí, 15 que respondieron todo No y dos personas que no respondieron.

\section{Motivación interna - MI}

Los ítems: se distribuyeron ocho por debajo y 10 por encima del promedio ubicado en 0 de la escala. El Infit por encima de $(2,0)$ corresponde a respuestas dadas de manera irreflexiva a ítems donde el grupo tenía habilidad para responderlos. Outfit dentro de los límites aceptados (Linacre indica que la medida por debajo de 0,5 no aporta a la medición, pero no la degrada, aunque puede alterar confiabilidad y separación $)^{34}$. Los ítems fueron fáciles para el grupo: el $56,7 \%$ obtuvo el máximo puntaje al responderlos. La confiabilidad estuvo en el límite aceptado y la separación permitió conformar tres estratos. 
Tabla 1. Resultados de parámetros Rasch por tipo de motivación según Deci y Ryan.

\begin{tabular}{|c|c|c|c|}
\hline Parámetros & Motivación Interna & Motivación Externa & Desmotivación \\
\hline $\mathrm{N}^{\circ}$ de Ítems & $\begin{array}{l}\text { Ítems } \\
18\end{array}$ & $\begin{array}{l}\text { Ítems } \\
17\end{array}$ & $\begin{array}{c}\text { Ítems } \\
13\end{array}$ \\
\hline Valores medida & $\begin{array}{c}-3.06-3.32 \text { prom } 0.0 \\
\text { DE } 2.11\end{array}$ & $\begin{array}{c}-4.10-4.18 \text { prom } 0.0 \\
\text { DE } 2.92\end{array}$ & $\begin{array}{c}-1.78-2.20 \text { prom } 0.0 \\
\text { DE } 1.0\end{array}$ \\
\hline Infit - MNSQ & $0.17-3.14$ prom 0.92 & $0.49-1.36$ prom 0.99 & $0.73-1.37$ prom 1.0 \\
\hline Outfit - MNSQ & $0.3-1.96$ prom 0.60 & $0.05-1.57$ prom 0.72 & $0.38-1.76$ prom 0.96 \\
\hline Confiabilidad & 0.80 & 0.97 & 0.89 \\
\hline Separación & 2.01 ( 3 estratos $)$ & 5.78 (8 estratos) & 2.83 (4 estratos) \\
\hline Unidimensionalidad & $\begin{array}{c}\text { Varianza por medidas } 41.2 \% \\
\text { Varianza inexplicada } 1 \mathrm{er} \\
\text { contraste } 4.0 \text { autovalores }\end{array}$ & $\begin{array}{l}\text { Varianza por medidas } 60.9 \% \\
\text { Varianza inexplicada } 1^{\mathrm{er}} \text { contraste } \\
2.3 \text { autovalores }\end{array}$ & $\begin{array}{c}\text { Varianza por medidas } 27.3 \% \\
\text { Varianza inexplicada } 1 \mathrm{er} \\
\text { contraste } 1.8 \text { autovalores }\end{array}$ \\
\hline $\mathrm{N}^{\circ}$ de Docentes & $\begin{array}{l}\text { Docentes } \\
104\end{array}$ & $\begin{array}{l}\text { Docentes } \\
101\end{array}$ & $\begin{array}{l}\text { Docentes } \\
102\end{array}$ \\
\hline Valores medida & $\begin{array}{c}-3.47-4.24 \text { prom } 3.03 \\
\text { DE } 1.59\end{array}$ & $-4.01-4.84$ prom 0.77 DE 1.44 & $\begin{array}{c}-2.87-2.00 \text { prom }-1.36 \mathrm{DE} \\
1.10\end{array}$ \\
\hline Infit - MNSQ & $0.61-1.66$ prom 1.0 & $0.22-2.69$ prom 1.0 & $0.50-1.61$ prom 1.0 \\
\hline Outfit - MNSQ & $0.19-2.86$ prom 0.60 & $0.08-3.66$ prom 0.71 & $0.25-2.58$ prom 0.96 \\
\hline Confiabilidad & 0.63 & 0.51 & 0.39 \\
\hline Separación & 1.29 ( 2 estratos) & 1.02 (1.7 estratos) & 0.79 (1.3 estratos) \\
\hline
\end{tabular}

Fuente: Elaboración propia - datos del estudio.

La unidimensionalidad corroborada por la varianza explicada por medidas fue buena con $41,2 \%$ (fuerte si es $\geq 40 \%$ según Linacre; moderada cuando es $\geq 30 \%$ según Conrad, et al. y mínima aceptable cuando es $\geq$ $20 \%$ según Reckase) $)^{35}$, pero la varianza inexplicada en el primer contraste estuvo una unidad sobre los 3,0 autovalores $^{36}$. La prueba de Mantel-Hanzel no encontró funcionamiento diferencial de ítems por sexo.

El grupo docente: rango de medida amplio, con promedio cercano al límite superior en la escala, por tanto, la mayoría (66\%) se ubicó en MI alta (Figura 2). El Infit mostró muy buen ajuste y su promedio en 1,0 indicó ajuste óptimo entre datos y modelo ${ }^{37}$; con relación al Outfil la medida superior por encima de 2,0 indicó respuestas al azar ante ítems donde no tenían habilidad. La confiabilidad, por debajo del mínimo aceptado $(0,80)$, estuvo afectada por falta de ítems para medir los niveles de habilidad altos donde se ubicó buena parte del profesorado ${ }^{38}$. La separación permitió dos estratos o grupos de docentes con diferente nivel de MI, siendo el mínimo aceptado tres estratos ${ }^{39}$. En el análisis de promedios de las medidas Rasch de profesores y variables sociodemográficas hubo diferencia estadísticamente significativa $(\mathrm{p}=0,03)$ en la variable motivos para ser docente observándose más MI en el grupo de personas que respondió "buscaba trabajo y salió esta oportunidad, no tenía más opciones" (Tabla 2).

\section{Motivación externa - ME}

Los ítems: mostraron ajuste en Infit y Outfit; la confiabilidad estuvo muy cercana al óptimo de 1,0 y la separación fue muy buena (ocho grupos con diferente nivel de dificultad). El 5\% del grupo respondió todos los ítems en forma correcta (máximo aceptado en Rasch).

El grupo docente: distribuido a lo largo del eje, excepto en el lógito más alto de la escala (Figura 2). En Infit y Outfit, se observaron límites superiores de las medidas por fuera del tope aceptado $(2,0)$ indicando respuestas al azar tanto en el terreno cercano (infit) como lejano (outfit) de sus habilidades relacionadas con ME; el promedio del infit muy cercano a 1,0 mostró buen ajuste, no así en el outfit. La confiabilidad fue baja y la separación mostró un solo grupo homogéneo en motivación externa. La unidimensionalidad se confirmó. 
Se encontró diferencia estadísticamente significativa por sexo en la ME: más motivados por razones externas, los hombres con casi un lógito de diferencia y quienes vivían en pareja (Tabla 2).

\section{Desmotivación - DESM}

La escala de medida en DESM fue más corta (-1,78 2,20); la mayoría de docentes se ubicó bajo el promedio de $-1,36$; los ítems se distribuyeron siete por debajo y seis sobre el promedio, pero dejando vacíos abajo y arriba de la escala (Figura 2).

En Infit y Outfit del grupo docente y de los ítems, hubo buen ajuste al modelo, aunque en el caso de docentes, el límite superior del Outfit orientó nuevamente a respuestas "por salir del paso" e ítems por construir para medir la alta desmotivación.
La confiabilidad en docentes fue bastante baja, influida por el déficit de ítems para medir alta desmotivación; la confiabilidad de los ítems fue buena. La separación mostró un solo grupo docente homogéneo en desmotivación en tanto que los ítems separaron cuatro grupos de diferente nivel de desmotivación.

La unidimensionalidad fue corroborada y ningún ítem tuvo FDI por sexo. En correlación de promedios hubo diferencias estadísticamente significativas: por sexo (menos DESM en mujeres, y menos DESM en mujeres con hijos menores de 18 años) y por título de pregrado siendo los más DESM los titulados en Agronomía, Veterinaria y afines y los menos DESM los del área de Ciencias sociales y humanas (Tabla 2).

Tabla 2. Relación de la MI, ME y DESM con variables sociodemográficas.

\begin{tabular}{|c|c|c|c|}
\hline Variables & Motivación interna & Motivación externa & Desmotivación \\
\hline Edad & No & No & No \\
\hline Sexo & No & $\begin{array}{l}\text { Más en hombres: } 1.55 . \text { Menos } \\
\text { en mujeres: } 0.63 . p=0.03\end{array}$ & $\begin{array}{c}\text { Más en hombres: }-.81 \text {. Menos en } \\
\text { mujeres: }-1.8 \mathrm{p}=0.008\end{array}$ \\
\hline Estado civil & No & $\begin{array}{l}\text { Más si vive en pareja: } 1.01 \text {. } \\
\text { Demás: } 0.10 . p=0.02\end{array}$ & No \\
\hline $\begin{array}{l}\text { Tener hijos } \\
<18 \text { años }\end{array}$ & No & No & $\begin{array}{c}\text { Más en hombres: }-1.33 \text {. Menos en } \\
\text { mujeres: }-1.98 . p=0.02\end{array}$ \\
\hline Otros menores a cargo & No & No & No \\
\hline Max nivel educativo & No & No & No \\
\hline Área conocimiento & No & No & $\begin{array}{l}\text { Más Agron, Veter y afi: } 1.3 \text {. } \\
\text { Menos Área Social: }-2.7 \mathrm{p}=0.003\end{array}$ \\
\hline Motivación docencia & $\begin{array}{l}\text { Si Grupo 3. p=0.03 } \\
\text { "buscaba trabajo y salió esta } \\
\text { oportunidad, no tenía más } \\
\text { opciones" }\end{array}$ & No & No \\
\hline Años experiencia & No & No & No \\
\hline Religión profesada & No & No & No \\
\hline
\end{tabular}

Fuente: Elaboración propia - datos del estudio.

\section{Etapa Cualitativa}

En el análisis de discurso de las entrevistas realizadas, se identificaron las motivaciones de tipo interno como las que mayormente se presentaron e influyeron en la respuesta a participar en el proceso formativo como una oportunidad para mejorar su práctica docente $\mathrm{y}$ aumentar sus conocimientos. Por otra parte, con relación a la motivación externa se reconoció que la participación está condicionada por el carácter normativo y obligatorio que tienen las instituciones educativas de incluir en sus currículos procesos de formación para la sexualidad. A continuación, se describen las dos categorías emergentes: Mejorar la práctica docente y Cumplimiento de normativas. 

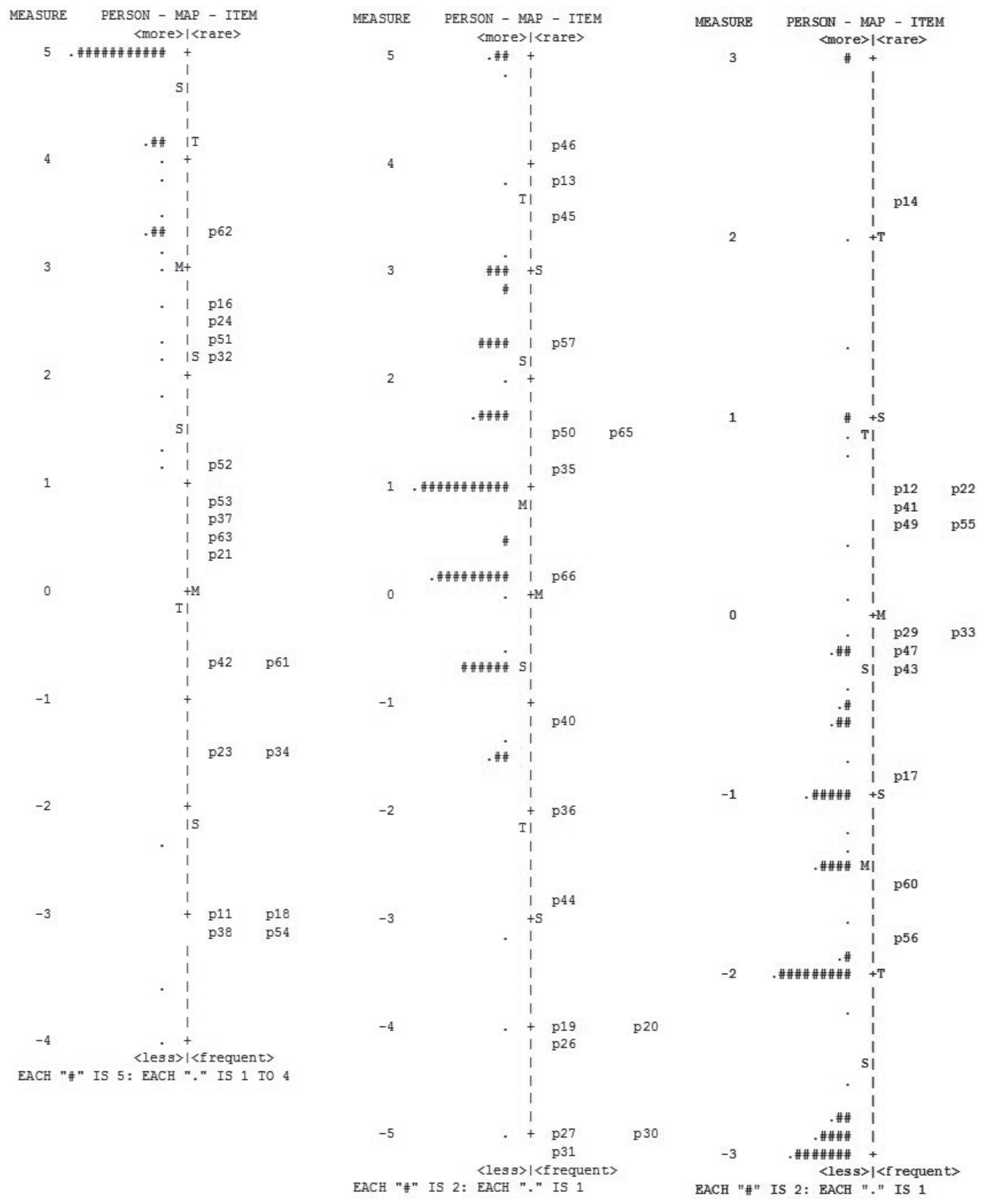

Figura 2. Mapas de Wrigh para motivación interna, externa y desmotivación.

Una oportunidad para mejorar nuestra práctica docente

Las personas docentes entrevistadas identificaron la participación en procesos de formación en sexualidad como una oportunidad para fortalecer y mejorar las experiencias pedagógicas y por ende su práctica docente; reconocieron que el contexto social en el que las y los jóvenes se desarrollan día a día, les obliga a contar con pautas mínimas que les permitan orientar a sus estudiantes en temas relacionados con salud sexual y reproductiva, "Empaparse uno del tema para llegar a 
los estudiantes de diferentes edades, mirar cómo puede uno ayudar de cierta manera y brindar información que facilite la vida del estudiante, que al fin y al cabo para eso es que estamos nosotros, para hacerles la vida como mejor" [Hombre, 43 años], "La sexualidad nos hace mucha falta, entenderla, saber qué es y para qué es" [Mujer, 43 años].

Así mismo, reconocieron en los procesos formativos una oportunidad para ampliar sus conocimientos sobre la temática, pese a los temores iniciales que generó el abordar un tema que ha sido tabú para la sociedad, "Porque es un tema que nosotros como docentes lo tenemos que manejar y lo tenemos que conocer, lo tenemos que estudiar. Porque es nuestro encuentro, nuestro diario vivir y en nuestra misma experiencia pedagógica en donde nos encontramos muchas veces con ciertas preguntas de los mismos estudiantes, muchas veces del padre de familia también, que no sabemos cómo manejar" [Mujer, 59 años], "Hablar de sexualidad eso es duro para mí como docente, pero uno necesita bases para poder hablar" [Hombre, 43 años], "La primera sensación es de impacto porque es un tema bastante dificil de manejar, no tenemos sabiduria sobre el tema entonces nos da susto porque se puede encaminar hacia cosas negativas, estábamos muy prevenidos" [Mujer, 32 años].

Estos conocimientos adquiridos, se constituyen en ayuda para mejorar su práctica docente y las relaciones con sus estudiantes, lo cual les permite acercarse de una forma diferente a sus realidades, "Pues cuando uno charla con ellos y uno dice, pero bueno, ¿yo cómo le puedo ayudar?, pues adquiriendo mayor conocimiento, así se les puede ayudar más" "Las ganas de aprender $y$ perder el miedo, ser capaz uno de hablar cuando un alumno le haga a uno una pregunta y ser capaz de contestar con bases" [Mujer, 59 años].

De la misma manera, un elemento que se reconoce, es el sentido dado por profesores a su labor docente, puesto que es reiterativa la asociación que se realiza entre su ejercicio pedagógico y la posibilidad de ayudar a mejorar las condiciones de vida de sus estudiantes, "Uno participa pues para ayudar a los chicos porque eso es lo que uno tiene que hacer... tratar de colaborar con los muchachos" [Mujer 43 años] "A los profesores nos gusta charlar con los estudiantes, colaborarles, apoyarlos...", "Esto es muy importante porque ayuda a uno a encaminar al alumno por un buen sendero" [Hombre 43 años].

\section{Cumplimiento de normas}

En el análisis de discurso se reconoció que la motivación para participar, está mediada también por factores externos relacionados con la exigencia dada por el Ministerio de Educación Nacional, quien definió de manera obligatoria la formación en educación sexual, mediante el Proyecto de Educación para la Sexualidad y Construcción de Ciudadanía, por tanto, el participar en procesos formativos sobre esta temática les permite dar respuesta a la normativa que regula su ejercicio docente, "Toca cumplir con el proyecto de educación sexual entonces con este proyecto, con la oportunidad estaba como facilito, se cumple de manera fácil" [Mujer, 52 años] "Imagínese, toca hacerle, porque si el Ministerio nos exige y si no estamos bien capacitados, qué les vamos a dar a los estudiantes?" [Mujer, 43 años].

De otra parte, el carácter de obligatoriedad definido por la autoridad municipal del sector educativo para participar en los procesos formativos, generó un rechazo inicial, lo cual está explicado por la sensación de asumir más carga laboral, participación en mesas de trabajo, horas adicionales de trabajo que implican menos tiempo para su vida personal y familiar; lo anterior afecta negativamente la motivación del personal docente, "Muchos lo tomamos con rechazo porque no sabíamos que tiempo nos iban a dar para eso" [Mujer, 47 años] "La palabra proyecto ya genera un cierto rechazo: no, mejor no... porque ya tenemos muchas cosas que hacer" [Mujer, 43 años] "Porque nosotros dijimos tras de que estamos de trabajo y ahora más trabajo, Tras de que no tenemos tiempo en el aula de clase y ahora hay que sacar más tiempo... " [Hombre 57 años].

Cabe mencionar que las personas clasificadas en la categoría de desmotivación, no asistieron a la fase de entrevistas por lo cual dichos aspectos no pudieron ser profundizados en esta etapa.

\section{Discusión}

Analizar las características de la motivación de un grupo de docentes de educación básica y media luego de recibir un proceso de formación en educación para la sexualidad y comprender desde su perspectiva las motivaciones que les llevaron a participar, fueron los objetivos del estudio; un reto importante pues la motivación es un constructo complejo en el contexto de la escuela, con las y los docentes como protagonistas: por ello se utilizó el diseño mixto a fin de lograr un análisis más integral ${ }^{40-42}$. 
Los resultados cuantitativos en las personas indicaron que la motivación interna fue la que tuvo mayor influencia en la participación docente (66\%) en el proceso formativo, aunque este resultado pudo tener algún nivel de imprecisión por la falta de ítems de alto nivel de dificultad, lo cual puede ser mejorado en próximos usos del instrumento. Las motivaciones intrínsecas tuvieron tiempo suficiente para ser estimuladas, pues el proceso formativo, permitió al grupo docente el ejercicio continuado de discutir, preguntar, reflexionar, replantear ideas y actitudes y mover sentimientos, necesidades, intereses y valores, asociados al trabajo formativo que han asumido con sus estudiantes; estos procesos internos conducirán a decisiones y compromisos duraderos referidos a su rol docente como lo plantean Deci y Ryan ${ }^{16}$. Este hallazgo sobre la motivación interna se ratificó desde el abordaje cualitativo, dado que, en el análisis de discurso de las entrevistas realizadas, se identificó claramente la formación en sexualidad como una oportunidad para mejorar su práctica docente y aumentar sus conocimientos, los cuales les permiten acercarse a la realidad de sus estudiantes y orientarlos de una mejor manera. Otros investigadores han encontrado resultados similares alrededor de la $\mathrm{MI}$, demostrándose que para motivar personas que han dedicado su vida a la docencia, los factores intrínsecos son los que mueven al compromiso y al mejoramiento. Por ello, lograr fortalecer la motivación en un grupo docente es un aporte significativo a la calidad de la educación, pues se ha encontrado evidencia de su relación con la autoeficacia, la satisfacción con el trabajo, el interés en asumir papeles adicionales en la escuela y la obtención de logros académicos importantes por sus estudiantes ${ }^{43-45}$.

La motivación externa tuvo los mejores resultados en ítems; en el análisis con variables sociodemográficas, los hombres fueron más sensibles a estímulos externos, lo cual coincidió con los resultados reportados por Balaguer, et al. ${ }^{46} \mathrm{y}$ otros tres estudios citados por este grupo de investigadores; la otra variable con significancia estadística fue el vivir en pareja: no se encontraron resultados ni coincidentes o contrarios en la bibliografía revisada pues esta variable no se incluyó en otros estudios. Al hacer las entrevistas a docentes se observó aceptación a la directriz del Ministerio de Educación de impartir educación sexual a sus estudiantes, una norma que no podían rechazar y lo más práctico era aprovechar la capacitación ofrecida, aunque inicialmente les preocupó, las horas adicionales que deberían aportar, en desmedro de su descanso y de su vida familiar. Al final del proceso formativo estas resistencias habían desaparecido, probablemente por las fuerzas motivacionales internas que se habían afianzado.
En el caso de la desmotivación - DESM, como se indicó, se dispone únicamente de la información cuantitativa; solo se encontró asociación entre DESM y sexo estando más desmotivados los hombres en general y aquellos que tiene hijos menores de 18 años; la otra variable que se asoció con DESM fue el área de conocimiento del título de pregrado donde las personas más desmotivadas fueron graduados de i) Agronomía, Veterinaria y afines, ii) Bellas Artes y iii) Economía, Administración, Contaduría y afines. Respecto a este punto, se comparte la reflexión de otros investigadores sobre la importancia de la formación para el ejercicio docente ya sea en pregrado o posgrado, en especial cuando se requiere una labor educativa incluyente $\mathrm{y}$ en contextos de alta vulnerabilidad social como son los que caracterizan la educación pública en los países de la región ${ }^{47-49}$; éste resultado también llama la atención sobre el Decreto 1278 de 2002 en Colombia donde se autoriza a profesionales con título diferente, legalmente habilitados para ejercer la profesión docente ${ }^{50}$ pues la idoneidad de la docencia se construye desde los procesos formativos específicos ${ }^{51}$ dado el requerimiento de competencias para la formación integral de estudiantes en sus dimensiones de personas, profesionales, ciudadanas y ciudadanos ${ }^{52}$.

Como conclusión se puede decir que esta investigación se presenta como pionera en el estudio de la motivación de docentes de educación básica y media en educación para la sexualidad, pues en la literatura consultada no se encontró ningún estudio con esta especificidad; sus resultados son un pequeño aporte para la capacitación y empoderamiento de docentes en el país y la región, a partir del estímulo de sus motivaciones internas para hacer educación para la sexualidad. Hay evidencia de que la autodeterminación se sintoniza muy bien con personas adultas que han elegido el rol docente para su vida laboral; además los procesos motivacionales en el escenario educativo tienen características propias según se trate de profesores o de estudiantes. Afortunadamente el interés en investigar en este campo se ha incrementado y hay estudios sobre motivación docente desde las diversas teorías existentes, sus fundamentos conceptuales y relaciones con el desempeño propio y de estudiantes para seguir avanzando en este campo del conocimiento ${ }^{27,53,54}$.

Desde el Instituto PROINAPSA UIS reconocemos la necesidad de continuar la labor de investigación, capacitación y apoyo a grupos docentes, quienes tienen el compromiso de orientar en las instituciones educativas, para que las nuevas generaciones logren vivir una sexualidad plena, respetuosa, libre $\mathrm{y}$ 
responsable en un marco de derechos que favorezca la construcción de sus proyectos de vida.

\section{Agradecimientos}

Agradecemos la colaboración del grupo docente que aceptó participar en el estudio. Nuestra profunda gratitud por siempre para el profesor Luis Carlos Orozco Vargas, quien apoyó el análisis y discusión de los resultados del trabajo.

\section{Consideraciones éticas}

De acuerdo con los principios establecidos en la Declaración de Helsinki y en la Resolución 8430 de octubre 4 de 1993 del Ministerio de Salud de Colombia, la presente investigación se clasificó como investigación sin riesgo.

\section{Referencias}

1. Hernández V. Las competencias emocionales del docente y su desempeño profesional. Alte Psicol. 2017; (37): 79-92.

2. Said-Hung E, Gratacós G, Valencia J. Factores que influyen en la elección de las carreras de pedagogía en Colombia. Educ. Pesqui, São Paulo. 2017; 43(1): 31-48. doi: http://dx.doi.org/10.1590/s15179702201701160978.

3. González Lara M. Paradojas en la formación docente: elementos para avanzar en su reflexión y planteamiento de propuestas. Colombia: Ediciones SM; Instituto para el Desarrollo y la Innovación Educativa IDIE - Formación de docentes y educadores. 2008. Bogotá.

4. Consejo Nacional de Política Económica y Social República de Colombia. Lineamientos para el desarrollo de una estrategia para la prevención del embarazo en la adolescencia y la promoción de proyectos de vida para los niños, niñas, adolescentes y jóvenes en edades entre 6 y 19 años. Documento CONPES Social 147. Bogotá, D.C. 2012.

5. Murillo F J, Krichesky G J. Mejora de la Escuela: Medio siglo de lecciones aprendidas. REICE. Revista Iberoamericana sobre Calidad, Eficacia y Cambio en Educación. 2014; 13(1): 69-102.

6. Colombia, Ministerio de Educación - UNFPA. Evaluación del programa de Educación para la sexualidad y construcción de ciudadanía - Informe Final. 2008. Bogotá.

7. Niño L, Hakspiel MC, Rincón AY, Aragón D, Roa ZM, Galvis DC et al. Cambios persistentes en conocimientos, actitudes y prácticas sobre sexualidad en adolescentes y jóvenes escolarizados de cuatro municipios de Santander. Rev Univ Ind Santander Salud. 2012; 44(2): 21-33.

8. Mantilla BP, Hakspiel MC, Rincón AY, Hernández DS, Hernández A. Promoción de derechos sexuales y reproductivos en adolescentes de Bucaramanga, Floridablanca y Lebrija - Colombia. Rev Univ Ind Santander Salud. 2013; 44(3): 13-23.

9. González Y. El papel del docente en la educación para la sexualidad: algunas reflexiones en el proceso educativo escolar. Rev Act Inv Edu. 2015; 15(3): 1-16. doi: http://dx.doi.org/10.15517/aie. v15i3.20335.

10. Fallas Vargas M A. Educación afectiva y sexual: programa de formación docentes de secundaria de Costa Rica (Tesis doctoral). Universidad de Salamanca. Departamento de Psicología Evolutiva y de la Educación. 2010.

11. Seixas Moizés J, Villela Bueno SM. Compreensão sobre sexualidade e sexo nas escolas segundo professores do ensino fundamental. Rev Esc Enferm USP. 2010; 44(1): 205-212. doi: http://dx.doi. org/10.1590/S0080-62342010000100029.

12. Caricote Agreda E. Los docentes y la educación sexual en la adolescencia. Rev Ciencias Edu. Valencia. 2008; 18(32): 13-33.

13. Kornblit AL, Sustas SE, Di Leo PF. Género, derechos sexuales, biografía y escuela: articulaciones y tensiones en discursos de docentes de Argentina. Educ Soc Campinas. 2014; 35(126): 161-178. doi: http://dx.doi.org/10.1590/S010173302014000100010.

14. Puchner L, Klein NA. Skirting the issue: Teachers' Experiences Addressing Sexuality in Middle School Language Arts. Rev RMLE. 2012; 36(1): 1-16.

15. Manassero M, Vásquez A. Análisis empírico de dos escalas de motivación escolar. Revista Electrónica de Motivación y Emoción. 2000; 3(5, 6): 1-20.

16. Deci EL, Ryan RM. The "what" and "why" of goal pursuits: human needs and the self-determination of behaviour. Psychol Inq. 2000; 11(4): 227-268.

17. Legaspi LP, Aisenson G, Valenzuela V, Duro L, De Marco M, Lavatelli L, et al. La motivación y el significado de la escuela para los jóvenes. Facultad de Psicología - Universidad Nacional de la Plata 2009.

18. Castillo I, Balaguer I, Duda JL. Propiedades psicométricas de la escala de motivación deportiva en deportistas españoles. Rev Mex Psicol. 2007; (24): 197-207.

19. Deci EL, Koestner R, Ryan RM. A meta-analytic review of experiments examining the effects of extrinsic rewards on intrinsic motivation. Psychol 
Bull. 1999; 125(6): 627-668.

20. Moreno JA, Martínez A. Importancia de la teoría de la autodeterminación en la práctica físico-deportiva: fundamentos e implicaciones prácticas. Cuad Psicol Deporte. 2006; 6(2): 39-54.

21. Vallerand R J, Blais M R, Brière N M, Pelletier LG. Construction et validation de l'Échelle de Motivation en Éducation (EME). Revue Canad Sci Comportement. 1989; (21): 323-349.

22. Millán M V. Factores de motivación relacionada con el aprendizaje en el estudiante de medicina. [Trabajo de grado para obtener el título de Médico Cirujano]. Universidad del Oriente. Barcelona, Venezuela. 2008.

23. Panadero, E, Tapia, JA. ¿Cómo autorregulan nuestros alumnos? Revisión del modelo cíclico de Zimmerman sobre autorregulación del aprendizaje. Anal Psicol. 2014; 30(2): 450-462.

24. Daura FT. Aprendizaje autorregulado y rendimiento académico en estudiantes del ciclo clínico de la carrera de Medicina. Rev Elect Invest Edu. 2015; 17(3).

25. Zarauz A, Ruiz-Juan F. Motivación, satisfacción, percepción y creencias sobre las causas del éxito en atletas veteranos españoles. Rev Iberoam Psicol Ej Deporte. 2016; 11(1): 37-46.

26. Vázquez SM, Daura F. Regulación, autodeterminación y libertad. Rev Logos Cienc Tecnol. 2013; 23: 69-89.

27. Carratalá E. Análisis de la teoría de las metas de logro y de la autodeterminación en los planes de especialización deportiva de la Generalitat Valenciana. Tesis Doctoral sin publicar, Facultad de Psicología, Universidad de Valencia, Valencia, España.2003.

28. Järvelä S, Järvenoja H. Socially Constructed SelfRegulated Learning and Motivation Regulation in Collaborative Learning Groups. Teachers College Record, Columbia University 2011. 113(2): 350374.

29. Creswell JW, Klassen AC, Plano Clark VI, Clegg Smith K. Best practices for mixed methods research in the health sciences. Bethesda (Maryland): National Institutes of Health. 2011; 2094-2103.

30. Valenzuela Medina JE. Formación de identidad en estudiantes universitarios: Un diseño combinado secuencial explicativo. [Tesis para obtener el grado de Doctor]. Universidad Autónoma de Sinaloa. Méjico, 2011.

31. Hakspiel-Plata MC, Niño-Bautista L, VelazcoRangel CM, Aragón-Borré D, Pineda-Rodríguez LP. Diseño y validación de encuesta sobre motivación de docentes frente a procesos de educación para la sexualidad. Rev Univ Ind Santander Salud. 2016; 18(1): 46-57.

32. Taylor SJ, Bogdan R. Introducción a los métodos cualitativos de investigación. La búsqueda de significados. Ediciones Paidos Iberica. 1987; 152160.

33. Jiménez K, Montero E. Aplicación del modelo de Rasch, en el análisis psicométrico de una prueba de diagnóstico en matemática. Revista digital Matemática, Educación e Internet. 2013; 13 (1). $1-24$.

34. Linacre J M. ¿What do In fit and Outfit, Meansquare and Standardized mean? Rasch Measurement Transactions. 2002, 16:2 p.878.

35. Meneses AL. Cuestionario de estrategias para la escritura de ensayos argumentativos. Acta Col Psicol. 2013; 16 (1): 137-148.

36. Bogoya D, Barragán S, Contento M, Ocaña A. Calibración de instrumentos de evaluación clasificación en matemáticas en la Universidad Jorge Tadeo Lozano. Rev Complutense Edu. 2014; 25 (2): 501-519. doi http://dx.doi.org/10.5209/rev_ RCED.2014.v25.n2.41931.

37. Delgado A R. La medida de la percepción emocional con el modelo de Rasch. Act Psicol. 2016; 30(120): 47-56. doi: http://dx.doi.org/10.15517/ ap.v29i119.21516.

38. Zamora JA. Análisis de la confiabilidad de los resultados de la prueba de diagnóstico matemática en la Universidad Nacional de Costa Rica utilizando el modelo de Rasch. Act Psicol. 2015; 29(119): 153-165. doi: http://dx.doi.org/10.15517/ ap.v29i119.18693.

39. Cupani M, Cortez D. Análisis psicométricos del subtest de razonamiento numérico utilizando el modelo de Rasch. Rev Psicol (Santiago). 2016; 25(2): 1-16.

40. Pereira Z. Los diseños de método mixto en la investigación en educación: una experiencia concreta. Rev Elect Educare. 2011; 15 (1): 15-29.

41. Guelmes E L, Nieto L E. Algunas reflexiones sobre el enfoque mixto de la investigación pedagógica en el contexto cubano. Rev Unive y Soc. 2015; 7 (1): 23-29.

42. Guerrero-Castaneda RF, Lenise do Prado M, OjedaVargas MG. Reflexión crítica epistemológica sobre métodos mixtos en investigación de enfermería. Enferm. Univ. 2016; 13(4): 246-252. doi: https:// doi.org/10.1016/j.reu.2016.09.001.

43. Buzdar MA, Mohsin MN, Akbar R, Mohammaduzda $\mathrm{N}$. Relationship between intrinsic motivation and students' academic achievement: a secondary level evidence. Bull Edu Res. 2017; 39 (2): 19-29. 
44. Covarrubias-Apablaza CG, Mendoza-Lira MC.

Educ Educ. 2016; 19 (3): 339-354. doi: 10.5294/ edu.2016.19.3.2.

45. Gomes CA, Palazzo J. Teaching career's attraction and rejection factors: analysis of students and graduate's perceptions in teacher education programs. Ensaio Aval Pol Públ Educ. 2017; 25 (94): 90-113. doi: http://dx.doi.org/10.1590/s010440362017000100004.

46. Balaguer I, Castillo I, Duda JL. Propiedades psicométricas de la escala de motivación deportiva en deportistas españoles. Rev Mexi Psicol. 2007; 24(2): 197-207.

47. Calvo G. La formación de docentes para la inclusión educativa. Pág Educ. 2013; 6 (1): 19-35.

48. Sánchez GI, Díaz SH, Fuentes AI, Osorio JB. Representaciones sobre el ejercicio de la docencia en contextos de vulnerabilidad de profesores de ciencias sociales en práctica. Pap Trabajo Cent Estud Interdiscip Etnolingüíst Antropol Soc Cult. 2017; (33): 64-82.

49. Beltrán JC. Factores que dificultan la gestión pedagógica curricular de los jefes de unidades técnico pedagógicas. Rev Mex Invest Edu. 2014; 19(62): 939-961.

50. Colombia. Decreto 1278 de junio 19 de 2002. Por el cual se expide el estatuto de profesionalización docente.

51. Cifuentes C. Impacto del Nuevo Estatuto de Profesionalización en la función docente en Colombia. Análisis de los dos estatutos vigentes: Decreto 2277 de 1979 y Decreto 1278 del 2002. Rev Col Sociol. 2014; 37(2): 213-250.

52. Galvis L A, Bonilla L. Desigualdades regionales en el nivel educativo de los profesores en Colombia. Rev.econ.inst. Bogotá. 2012; 14 (26): 223-240.

53. Han J, Yin H. Teacher motivation: Definition, research development and implications for teachers. Cogent Education. 2016; (3): 1-18. doi: http:// dx.doi.org/10.1080/2331186X.2016.1217819.

54. Sosic-Vasic Z, Keis O, Lau M, Spitzer M, Streb J. The impact of motivation and teachers' autonomy support on children's executive functions. Front Psychol. 2015; (6): 146. doi: 10.3389/ fpsyg.2015.00146. 